\title{
Periodically-Focused Solutions to the Nonlinear Vlasov-Maxwell Equations for Intense Beam Propagation Through an Alternating-Gradient Quadrupole Field
}

\author{
Hong Qin and Ronald C. Davidson \\ Plasma Physics Laboratory \\ Princeton University, Princeton, NJ, 08543 \\ Paul J. Channell \\ Los Alamos National Laboratory \\ Los Alamos, NM 87545
}

\begin{abstract}
This paper considers an intense nonneutral ion beam propagating in the $z$-direction through a periodic focusing quadrupole field with transverse focusing force, $\mathbf{F}_{f o c}=-\kappa_{q}(s)\left(x \hat{\mathbf{e}}_{x}-y \hat{\mathbf{e}}_{y}\right)$, on the beam ions. A third-order Hamiltonian averaging technique using a canonical transformation is employed to transform away the rapidly oscillating terms. This leads to a Hamiltonian, $\mathcal{H}\left(\tilde{X}, \tilde{Y}, \tilde{X}^{\prime}, \tilde{Y}^{\prime}, s\right)=(1 / 2)\left(\tilde{X}^{\prime 2}+\tilde{Y}^{\prime 2}\right)+$ $(1 / 2) \kappa_{f q}\left(\tilde{X}^{2}+\tilde{Y}^{2}\right)+\psi(\tilde{X}, \tilde{Y}, s)$, in the transformed variables $\left(\tilde{X}, \tilde{Y}, \tilde{X}^{\prime}, \tilde{Y}^{\prime}\right)$, where the focusing coefficient $\kappa_{f q}$ is constant, and many solutions and properties of the VlasovMaxwell system are well known.
\end{abstract}

\section{INTRODUCTION}

It is important to be able to investigate, based on the nonlinear Vlasov-Maxwell equations, the equilibrium and stability properties of general distribution functions for periodically-focused beams[1, 2, 3]. Despite its limited practical interest due to the unphysical distribution in phase space, the Kapchinskij-Vladimirskij (KV) beam equilibrium[1, 4, 5, 6], including its recent generalization to a rotating beam in a periodic focusing solenoidal field[7, 8], has been the only known periodically-focused equilibrium solution to the nonlinear Vlasov-Maxwell equations describing an intense beam propagating through a periodic focusing field. The difficulty of solving the nonlinear Vlasov-Maxwell system in general lies in the fact that the Hamiltonian for the motion of an individual beam particle is time dependent. Channell[9] and Davidson et al[10] have recently developed a third-order Hamiltonian averaging technique using a canonical transformation to average over the fast time scale associated with the betatron oscillations. This procedure is expected to be valid for sufficiently small phase advance $\left(\sigma \lesssim 60^{\circ}\right.$, say). In the present analysis, we apply this technique to the Vlasov-Maxwell system for intense beams propagating through a periodic focusing lattice. Under the thinbeam assumption, the applied transverse focusing force on a beam particle is $\mathbf{F}_{f o c}=-\kappa_{q}(s)\left(x \hat{\mathbf{e}}_{x}-y \hat{\mathbf{e}}_{y}\right)$. The Vlasov-Maxwell equations for the distribution function $f_{b}\left(x, y, x^{\prime}, y^{\prime}, s\right)$ and the normalized self-field potential $\psi(x, y, s)=Z_{b} e \phi(x, y, s) / \gamma_{b}^{3} m_{b} \beta_{b}^{2} c^{2}$ can be expressed as $[1,7]$

$$
\begin{aligned}
& \left\{\frac{\partial}{\partial s}+x^{\prime} \frac{\partial}{\partial x}+y^{\prime} \frac{\partial}{\partial y}-\left(\kappa_{q}(s) x+\frac{\partial \psi}{\partial x}\right) \frac{\partial}{\partial x^{\prime}}\right. \\
& \left.-\left(-\kappa_{q}(s) y+\frac{\partial \psi}{\partial y}\right) \frac{\partial}{\partial y^{\prime}}\right\} f_{b}=0,
\end{aligned}
$$

and

$$
\left(\frac{\partial^{2}}{\partial x^{2}}+\frac{\partial^{2}}{\partial y^{2}}\right) \psi=-\frac{2 \pi K_{b}}{N_{b}} \int d x^{\prime} d y^{\prime} f_{b} .
$$

Here,

$$
K_{b}=\frac{2 N_{b} Z_{b}^{2} e^{2}}{\gamma_{b}^{3} m_{b} \beta_{b}^{2} c^{2}} \text { and } N_{b}=\int d x d y d x^{\prime} d y^{\prime} f_{b}
$$

are the self-field perveance and the number of beam ions per unit axial length, respectively.

\section{CANONICAL TRANSFORMATION}

Because of the oscillatory time dependence of $\kappa_{q}(s)$, there is no general analytical method to solve the nonlinear Vlasov-Maxwell equations. However, we can average over the fast time scale associated with the betatron oscillations when the phase advance is sufficiently small. The averaging process is accomplished by introducing a canonical coordinate transformation from the laboratory coordinate system $\left(x, y, x^{\prime}, y^{\prime}\right)$ to a new coordinate system $\left(X, Y, X^{\prime}, Y^{\prime}\right)$. In the laboratory coordinates, the singleparticle Hamiltonian $H\left(x, y, x^{\prime}, y^{\prime}, s\right)$ is

$H=\epsilon\left[\frac{1}{2}\left(x^{\prime 2}+y^{\prime 2}\right)+\frac{1}{2} \kappa_{q}(s)\left(x^{2}-y^{2}\right)+\psi(x, y, s)\right]$,

where $\epsilon$ is a small dimensionless parameter proportional to the focusing field strength. We use a near-identity canonical transformation $T:\left(x, y, x^{\prime}, y^{\prime}\right) \longmapsto\left(X, Y, X^{\prime}, Y^{\prime}\right)$ that is generated by a generating function of the Von Zeipel form, i.e.,

$$
\begin{aligned}
& S\left(x, y, X^{\prime}, Y^{\prime}, s\right)=x X^{\prime}+y Y^{\prime} \\
& +\sum_{n=1}^{\infty} \epsilon^{n} S_{n}\left(x, y, X^{\prime}, Y^{\prime}, s\right) .
\end{aligned}
$$


Consequently, the transformed Hamiltonian in the new variables $\mathcal{H}\left(\tilde{X}, \tilde{Y}, \tilde{X}^{\prime}, \tilde{Y}^{\prime}, s\right)$ is given by

$$
\mathcal{H}=\sum_{n=1}^{\infty} \epsilon^{n} \mathcal{H}_{n}=H+\frac{\partial}{\partial s} S\left(x, y, X^{\prime}, Y^{\prime}, s\right) \text {. }
$$

The corresponding coordinate transformation is given by

$$
\begin{aligned}
& X=\frac{\partial S}{\partial X^{\prime}}=x+\sum_{n=1}^{\infty} \epsilon^{n} \frac{\partial}{\partial X^{\prime}} S_{n}\left(x, y, X^{\prime}, Y^{\prime}, s\right) \\
& x^{\prime}=\frac{\partial S}{\partial x}=X^{\prime}+\sum_{n=1}^{\infty} \epsilon^{n} \frac{\partial}{\partial x} S_{n}\left(x, y, X^{\prime}, Y^{\prime}, s\right) .
\end{aligned}
$$

The equations for $Y$ and $y^{\prime}$ are similar in form.. We choose, order by order, the generating function $S_{n}$ in such a way that $\mathcal{H}_{n}$ is independent of the fast time scale associated with oscillations in $\kappa_{q}(s)$, and solve for the coordinate transformation iteratively when $S_{n}$ is known. Following the detailed algebra presented in Ref. [10], we obtain the transformed Hamiltonian correct to order $\epsilon^{3}$,

$$
\mathcal{H}=\frac{1}{2}\left(\tilde{X}^{\prime 2}+\tilde{Y}^{\prime 2}\right)+\frac{1}{2} \kappa_{f q}\left(\tilde{X}^{2}+\tilde{Y}^{2}\right)+\psi(\tilde{X}, \tilde{Y}, s)
$$

where we have set $\epsilon=1$. Here, $\kappa_{f q}$ is defined in Eq. (11), and we have introduced the additional (canonical) fiber transformation to shifted velocity coordinates defined by

$$
\begin{aligned}
& \tilde{X}=X, \quad \tilde{X}^{\prime}=X^{\prime}-\left\langle\alpha_{q}\right\rangle X, \\
& \tilde{Y}=Y, \quad \tilde{Y}^{\prime}=Y^{\prime}+\left\langle\alpha_{q}\right\rangle Y .
\end{aligned}
$$

Similarly, correct to order $\epsilon^{3}$, we calculate the inverse coordinate transformation, $x=X+\epsilon x_{1}+\epsilon^{2} x_{2}+\epsilon^{3} x_{3}$, $x^{\prime}=X^{\prime}+\epsilon x_{1}^{\prime}+\epsilon^{2} x_{2}^{\prime}+\epsilon^{3} x_{3}^{\prime}$, etc. Setting $\epsilon=1$, this gives[10]

$$
\begin{aligned}
& x\left(\tilde{X}, \tilde{Y}, \tilde{X}^{\prime}, \tilde{Y}^{\prime}, s\right)=\left[1-\beta_{q}(s)\right] \tilde{X}+2\left(\int_{0}^{s} d s \beta_{q}(s)\right) \tilde{X}^{\prime}, \\
& x^{\prime}\left(\tilde{X}, \tilde{Y}, \tilde{X}^{\prime}, \tilde{Y}^{\prime}, s\right)=\left[1+\beta_{q}(s)\right] \tilde{X}^{\prime}+\left\{-\alpha_{q}(s)+\left\langle\alpha_{q}\right\rangle\right. \\
& \left.+\left\langle\alpha_{q}\right\rangle \beta_{q}(s)-\alpha_{q}(s) \beta_{q}(s)-\left(\int_{0}^{s} d s\left[\delta_{q}(s)-\left\langle\delta_{q}\right\rangle\right]\right)\right\} \tilde{X} \\
& +\left(\int_{0}^{s} d s \beta_{q}(s)\right) \frac{\partial}{\partial \tilde{X}}\left(\tilde{X} \frac{\partial \psi(\tilde{X}, \tilde{Y})}{\partial \tilde{X}}-\tilde{Y} \frac{\partial \psi(\tilde{X}, \tilde{Y})}{\partial \tilde{Y}}\right) .
\end{aligned}
$$

The coordinate transformation can be easily obtained by solving Eq. (10) for $\tilde{X}$ and $\tilde{X}^{\prime}$ in terms of $x$ and $x^{\prime}$. The expressions for $y$ and $y^{\prime}$ are identical in form to Eq. (10) provided we make the replacements $\left(x, x^{\prime}\right) \rightarrow\left(y, y^{\prime}\right)$ and $\left(\tilde{X}, \tilde{Y}, \tilde{X}^{\prime}, \tilde{Y}^{\prime}\right) \rightarrow\left(\tilde{Y}, \tilde{X}, \tilde{Y}^{\prime}, \tilde{X}^{\prime}\right)$ and reverse the signs of $\alpha_{q}(s)$ and $\beta_{q}(s)$. In the above equations, $\alpha_{q}(s), \beta_{q}(s)$, and $\delta_{q}(s)$ are defined in terms of the lattice function $\kappa_{q}(s)$, which is assumed to have zero average, $\int_{0}^{S} d s \kappa_{q}(s)=$
0 , and odd half-period symmetry with $\kappa_{q}(s-S / 2)=$ $-\kappa_{q}[-(s-S / 2)]$. The definitions are given by

$$
\begin{aligned}
& \alpha_{q}(s)=\int_{0}^{s} d s \kappa_{q}(s), \beta_{q}(s)=\frac{1}{S} \int_{0}^{s} d s\left[\alpha_{q}(s)-\left\langle\alpha_{q}\right\rangle\right], \\
& \langle\ldots\rangle \equiv \frac{1}{S} \int_{0}^{S} d s(\ldots), \delta_{q}(s)=\alpha_{q}^{2}(s)-2 \kappa_{q}(s) \beta_{q}(s), \\
& \kappa_{f q}=\left\langle\delta_{q}\right\rangle-\left\langle\alpha_{q}\right\rangle^{2}=\frac{3}{S} \int_{0}^{S} d s\left[\alpha_{q}^{2}(s)-\left\langle\alpha_{q}\right\rangle^{2}\right] .
\end{aligned}
$$

In addition, $\alpha_{q}(s)$ and $\left\langle\alpha_{q}\right\rangle$ are of order $\epsilon ; \beta_{q}(s)$ is of order $\epsilon^{2}$; and $\left\langle\alpha_{q}\right\rangle \beta_{q}(s), \alpha_{q}(s) \beta_{q}(s),\left(\int_{0}^{s} d s \beta_{q}(s)\right)$, and $\left(\int_{0}^{s} d s\left[\delta_{q}(s)-\left\langle\delta_{q}\right\rangle\right]\right)$ are of order $\epsilon^{3}$.

\section{VLASOV-MAXWELL EQUATIONS IN THE TRANSFORMED VARIABLES}

Because the transformation leading to the new Hamiltonian in Eq. (8) is canonical, the nonlinear Vlasov-Maxwell equations for the distribution function $F_{b}\left(\tilde{X}, \tilde{Y}, \tilde{X}^{\prime}, \tilde{Y}^{\prime}, s\right)$ and self-field potential $\psi(\tilde{X}, \tilde{Y}, s)$ in the transformed variables are given by

$$
\begin{aligned}
& \left\{\frac{\partial}{\partial s}+\tilde{X}^{\prime} \frac{\partial}{\partial \tilde{X}}+\tilde{Y}^{\prime} \frac{\partial}{\partial \tilde{Y}}-\left(\kappa_{f q} \tilde{X}+\frac{\partial \psi}{\partial \tilde{X}}\right) \frac{\partial}{\partial \tilde{X}^{\prime}}\right. \\
& \left.-\left(\kappa_{f q} \tilde{Y}+\frac{\partial \psi}{\partial \tilde{Y}}\right) \frac{\partial}{\partial \tilde{Y}^{\prime}}\right\} F_{b}=0,
\end{aligned}
$$

and

$$
\left(\frac{\partial^{2}}{\partial \tilde{X}^{2}}+\frac{\partial^{2}}{\partial \tilde{Y}^{2}}\right) \psi=-\frac{2 \pi K_{b}}{N_{b}} \int d \tilde{X}^{\prime} d \tilde{Y}^{\prime} F_{b},
$$

where $\kappa_{f q}=$ const. is defined in Eq. (11). Variables in laboratory-frame coordinates can be obtained through the pull-back transformation $\tilde{T}^{*}$ associated with the coordinate transformation

$$
\tilde{T}:\left(x, y, x^{\prime}, y^{\prime}\right) \longmapsto\left(\tilde{X}, \tilde{Y}, \tilde{X}^{\prime}, \tilde{Y}^{\prime}\right) .
$$

Here, $\tilde{T}^{*}$ pulls (transforms) functions on $\left(\tilde{X}, \tilde{Y}, \tilde{X}^{\prime}, \tilde{Y}^{\prime}\right)$ back into functions on $\left(x, y, x^{\prime}, y^{\prime}\right)$. For example, the distribution function transforms according to

$$
\begin{aligned}
& \tilde{T}^{*}: F_{b}\left(\tilde{X}, \tilde{Y}, \tilde{X}^{\prime}, \tilde{Y}^{\prime}, s\right) \longmapsto f_{b}\left(x, y, x^{\prime}, y^{\prime}, s\right) \\
& \equiv F_{b}\left(\tilde{T}\left(x, y, x^{\prime}, y^{\prime}\right), s\right)
\end{aligned}
$$

In addition, we obtain the following pull-back equation for the beam density correct to order $\epsilon^{3}$,

$$
\begin{aligned}
& n_{b}(x, y, s)=\int d \bar{x} d \bar{y} d x^{\prime} d y^{\prime} f_{b} \delta(\bar{x}-x) \delta(\bar{y}-y) \\
& =\int d \tilde{X} d \tilde{Y} d \tilde{X}^{\prime} d \tilde{Y}^{\prime} F_{b} \delta\left(\tilde{T}^{-1} \tilde{X}-x\right) \delta\left(\tilde{T}^{-1} \tilde{Y}-y\right) \\
& =\left\{\int d \tilde { X } ^ { \prime } d \tilde { Y } ^ { \prime } \left[1-\left(x_{2}+x_{3}\right) \frac{\partial}{\partial \tilde{X}}\right.\right. \\
& \left.\left.-\left(y_{2}+y_{3}\right) \frac{\partial}{\partial \tilde{Y}}\right] F\right\}_{(\tilde{X}, \tilde{Y}) \rightarrow(x, y)} .
\end{aligned}
$$


Here, $x_{2}, y_{2}$ and $x_{3}, y_{3}$, defined by Eq. (10), are the second-order and third-order inverse coordinate transformations expressed as functions of $\left(\tilde{X}, \tilde{Y}, \tilde{X}^{\prime}, \tilde{Y}^{\prime}\right)$.

Because of the simple form of the Vlasov-Maxwell equations in the transformed variables, with constant focusing coefficient $\kappa_{f q}=$ const., a wide range of literature developed for the constant focusing case[1, 11, 12, 13] can be applied virtually intact in the transformed variables. For example, it is readily shown that any distribution function of the form

$$
F_{b}^{0}\left(\tilde{X}, \tilde{Y}, \tilde{X}^{\prime}, \tilde{Y}^{\prime}\right)=F_{b}^{0}\left(\mathcal{H}^{0}\right)
$$

where $\mathcal{H}^{0}=(1 / 2)\left(\tilde{X}^{\prime 2}+\tilde{Y}^{\prime 2}\right)+(1 / 2) \kappa_{f q}\left(\tilde{X}^{2}+\tilde{Y}^{2}\right)+$ $\psi^{0}(\tilde{X}, \tilde{Y})$ is the single-particle Hamiltonian, is an exact equilibrium solution to the Vlasov-Maxwell equations (12) and (13) with $\partial / \partial s=0$. There is clearly enormous latitude[1, 7] in specifying the functional form of $F_{b}^{0}\left(\mathcal{H}^{0}\right)$ in the transformed variables, with equilibrium examples[10] ranging from the KV distribution, to the waterbag equilibrium, to thermal equilibrium, to mention a few examples. Once the functional form of $F_{b}^{0}\left(\mathcal{H}^{0}\right)$ is specified, and $\psi^{0}$ is calculated self-consistently from Eq. (13), periodically-focused equilibrium properties in the laboratory coordinates, such as the density profile and the transverse temperature profile, can then be determined by the pull-back transformation. For example, to the leading order, the density profile is of the form[10]

$$
n_{b}(x, y, s)=n_{b}^{0}\left(\frac{x}{1-\beta_{q}(s)}, \frac{y}{1+\beta_{q}(s)}\right),
$$

where $n_{b}^{0}(\tilde{X}, \tilde{Y})=\int d \tilde{X}^{\prime} d \tilde{Y}^{\prime} F_{b}^{0}\left(\tilde{X}, \tilde{Y}, \tilde{X}^{\prime}, \tilde{Y}^{\prime}\right)$.

\section{CONCLUSIONS}

To summarize, the formalism developed here represents a powerful framework for investigating the kinetic equilibrium and stability properties of an intense nonneutral ion beam propagating through an alternating-gradient quadrupole field. First, the analysis applies to a broad class of equilibrium distributions $F_{b}^{0}\left(\mathcal{H}^{0}\right)$ in the transformed variables. Second, the determination of (periodicallyfocused) beam properties in the laboratory frame is relatively straightforward. Third, the analysis applies to beams with arbitrary space-charge intensity, consistent only with requirement for radial confinement of the beam particles by the applied focusing field $\left(\kappa_{f q} \beta_{b}^{2} c^{2}>\hat{\omega}_{p b}^{2} / 2 \gamma_{b}^{2}\right)$. Finally, the formalism can be extended[10] in a straightforward manner to the case of a periodic-focusing solenoidal field $\mathbf{B}_{s o l}(\mathbf{x})=B_{z}(s) \hat{\mathbf{e}}_{z}-(1 / 2) B_{z}^{\prime}(s)\left(x \hat{\mathbf{e}}_{x}+y \hat{\mathbf{e}}_{y}\right)$, and to the case where weak nonlinear corrections to the focusing force are retained in the analysis.

\section{ACKNOWLEGEMENT}

This research was supported by the Department of Energy.

\section{REFERENCES}

[1] R. C. Davidson, Physics of Nonneutral Plasmas (AddisonWesley Publishing Co., Reading, MA, 1990), and references therein.

[2] T. P. Wangler, Principles of RF Linear Accelerators (John Wiley \& Sons, Inc., New York, 1998).

[3] M. Reiser, Theory and Design of Charged Particle Beams (John Wiley \& Sons, Inc., New York, 1994).

[4] I. Kapchinskij and V. Vladimirskij, in Proceedings of the International Conference on High Energy Accelerators and Instrumentation (CERN Scientific Information Service, Geneva, 1959), p. 274.

[5] R. L. Gluckstern, in Proceedings of the 1970 Proton Linear Accelerator Conference, Batavia, IL, edited by M. R. Tracy (National Accelerator Laboratory, Batavia, IL, 1971), p. 811.

[6] T. -S. Wang and L. Smith, Particle Accelerators 12, 247 (1982).

[7] R. C. Davidson and C. Chen, Particle Accelerators 59, 175 (1998).

[8] C. Chen, R. Pakter, and R. C. Davidson, Phys. Rev. Lett. 79, 225 (1997).

[9] P. J. Channell, Physics of Plasmas 6, 982 (1999).

[10] R. C. Davidson, H. Qin, and P. J. Channell, to be published (1999).

[11] R. C. Davidson, W. W. Lee, and P. H. Stoltz, Phys. Plasmas 5, 279 (1998).

[12] R. C. Davidson, Physical Review Letters 81, 991 (1998).

[13] R. C. Davidson, Physics of Plasmas 5, 3459 (1998). 